\title{
Defect and strain in hydrogen and helium coimplanted single-crystal silicon
}

\author{
Xinzhong Duo ${ }^{1}$, Weili Liu ${ }^{1}$, Su Xing ${ }^{1}$, Miao Zhang ${ }^{1}$, Xiaorong Fu ${ }^{1}$, \\ Chenglu Lin ${ }^{1}$, Pegang Hu${ }^{2}$, S X Wang ${ }^{3}$ and L M Wang ${ }^{3}$ \\ ${ }^{1}$ State Key Laboratory of Functional Materials for Informatics, \\ Shanghai Institute of Metallurgy, Chinese Academy of Sciences, Shanghai 200050, \\ People's Republic of China \\ 2 Applied Ion Beam Physics Laboratory, Fudan University, Shanghai 200433, \\ People's Republic of China \\ ${ }^{3}$ Department of Nuclear Engineering and Radiological Sciences, College of Engineering, \\ The University of Michigan, MI, USA
}

Received 7 July 2000, in final form 19 September 2000

\begin{abstract}
In this paper we studied the processes of blistering and exfoliation on the surface of crystal silicon, the evolution of defects/strains in the crystal silicon caused by hydrogen and helium coimplantation during annealing, and the formation of platelets and bubbles in the crystal. It is shown that $\mathrm{H}^{+}$ and $\mathrm{He}^{+}$coimplantation produces a synergistic effect, which greatly decreases the total implantation dose, compared with either just $\mathrm{H}^{+}$or $\mathrm{He}^{+}$ implantation. We also present the effect of coimplantation and analyse the different roles of $\mathrm{H}$ and $\mathrm{He}$ in the process of exfoliation during annealing. It seems that the essential role of hydrogen is to interact chemically with the defects in the silicon and create $\mathrm{H}$-stabilized platelets, while the role of helium is to effuse into these platelets and exert a pressure on the inner surface of these platelets. The damage caused by coimplantation is lower than by hydrogen implantation (at the dose that exfoliation requires).
\end{abstract}

\section{Introduction}

The physics and chemistry of hydrogen in silicon have been the subjects of considerable scientific and technological interest for decades. Hydrogen profoundly alters the electrical characteristics of a resultant device by diffusing into the active region and passivating the dopant [1-3]. Furthermore, hydrogen also causes surface exfoliation and blistering under certain conditions [4-6]. At present, there is great interest focusing on thin-film separation in the smart-cut process (one of the most important steps in Unibond silicon on insulator (SOI) material production), which is based on the phenomenon of implanted hydrogen or helium causing exfoliation and blistering on the surface [7]. This process allows SOI wafers to be produced more economically and with better quality than competing processes such as SIMOX (separated by implantation oxygen) and BESOI (bonded and etch-back SOI). Gas ion implantation, usually hydrogen, is a necessary step in the smart-cut process. Generally, it is considered that the thin-film separation process is induced by chemical interaction (bond breaking and internal surface passivation) and physical interaction (gas coalescence, pressure and fracture) of the implanted $\mathrm{H}$ in the silicon substrate. It is difficult to isolate the contribution of each component to the overall process. Helium is another kind of gas used in thin-film separation. Helium does not chemically interact with the silicon atoms in an $\mathrm{H}$-implanted substrate, but physically interacts in the substrate just as hydrogen does [8]. The coimplantation of hydrogen and helium is a good way in which to study the different interactions of implanted gas ions in implanted samples [9]. Implanted ions help to decouple the physical and chemical contributions to the blistering and thinfilm separation processes, and to understand both interactions of hydrogen in crystal silicon and compare the different roles of hydrogen and helium in the samples. Coimplantation requires a much lower total dose of ion implantation than the dose required for hydrogen ion implantation alone. Coimplantation will shorten the implantation time and decrease crystal damage during implantation, which then decreases the cost and improves the quality of the smart-cut SOI. In this work, high-resolution x-ray diffractometry (HRXRD), Rutherfordbackscattering spectrometry and channelling (RBS/C), elastic recoil detection analysis (ERDA), cross sectional transmission electron microscopy (XTEM), atom force microscopy (AFM) 


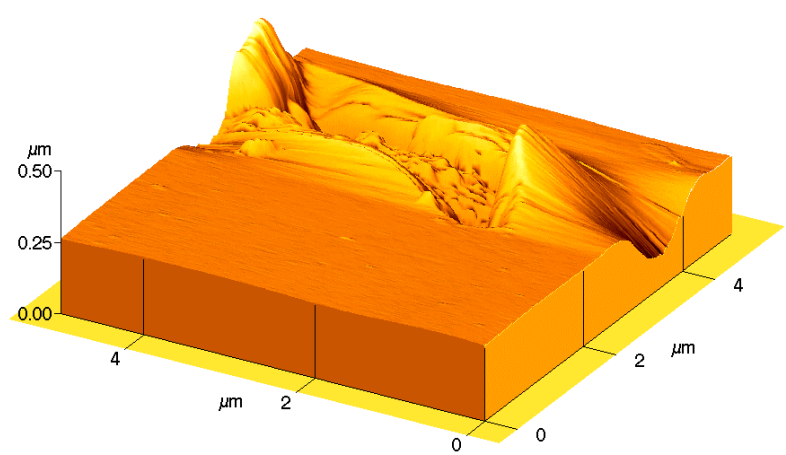

(a)

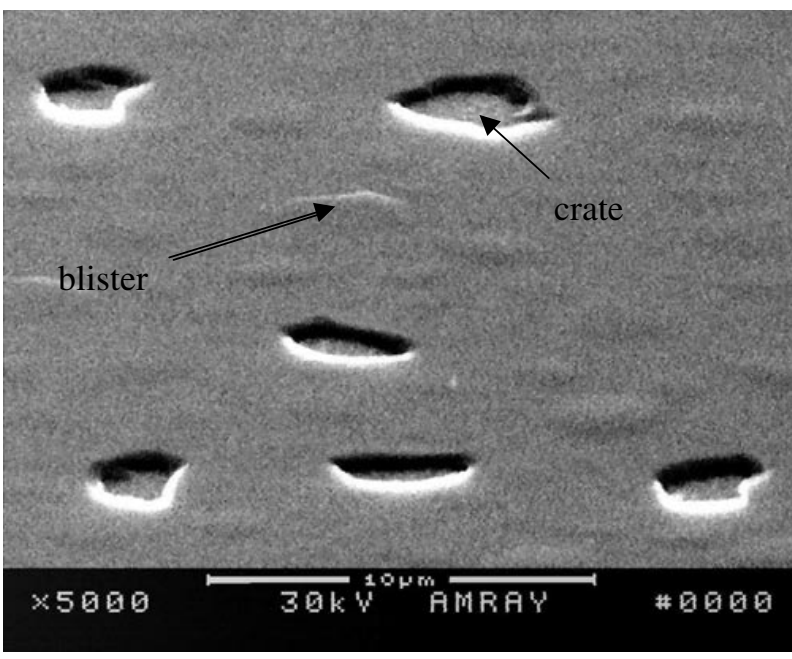

(b)

Figure 1. Surface images of a sample annealed at $450{ }^{\circ} \mathrm{C}$ for $1 \mathrm{~h}$. (a) AFM micrograph of the surface showing a crater on the surface formed after exfoliation. (b) A SEM photomicrograph of the blisters and craters on the surface after annealing.

and scanning electron microscopy (SEM) are used to study the coimplanted samples prepared at different annealing temperatures.

\section{Experimental details}

Wafers of p-type (5-8 $\Omega \mathrm{cm}$ ) Czochralski grown (100) Si were implanted at room temperature with $\mathrm{H}^{+}$at $30 \mathrm{keV}$ to a dose of $1 \times 10^{16} \mathrm{~cm}^{2}$ and then $\mathrm{He}^{+}$at $33 \mathrm{keV}$ to a dose of $1 \times 10^{16} \mathrm{~cm}^{2}$. In order to avoid channelling effects, a deliberate misalignment from the wafer normal of $7^{\circ}$ was used during implantation. After implantation, all wafers were cut into smaller samples for analysis. The samples were given a standard RCA clean prior to analysis in order to remove surface contaminants introduced by the implantation process. They were then annealed in a temperature range of $200-450^{\circ} \mathrm{C}$ for $1 \mathrm{~h}$ in a flowing ambient of $\mathrm{N}_{2}$ gas.

HRXRD was performed on a Philips X'pert equipped with a two-crystal four-reflection Ge [220] diffractometer. $\mathrm{Cu} \mathrm{K} \alpha_{1}$ radiation with a wavelength of $1.5406 \AA$ and fourfold $\mathrm{Ge}$ (220) reflection were used in all the experiments. An $\omega / 2 \theta$ scan was performed on the working plane (004). The curves were computer simulated and as a result the profiles

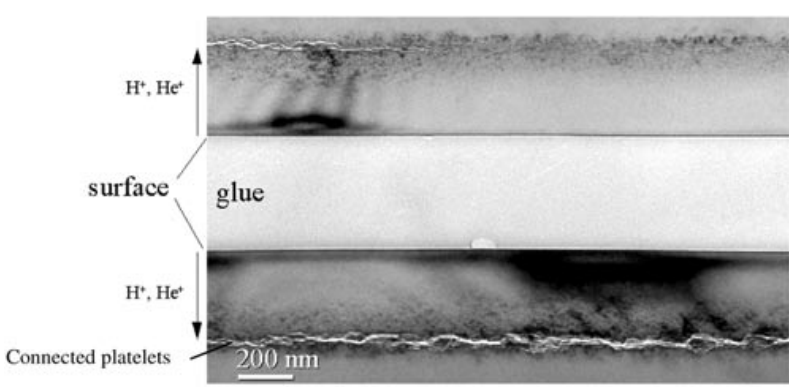

(a)

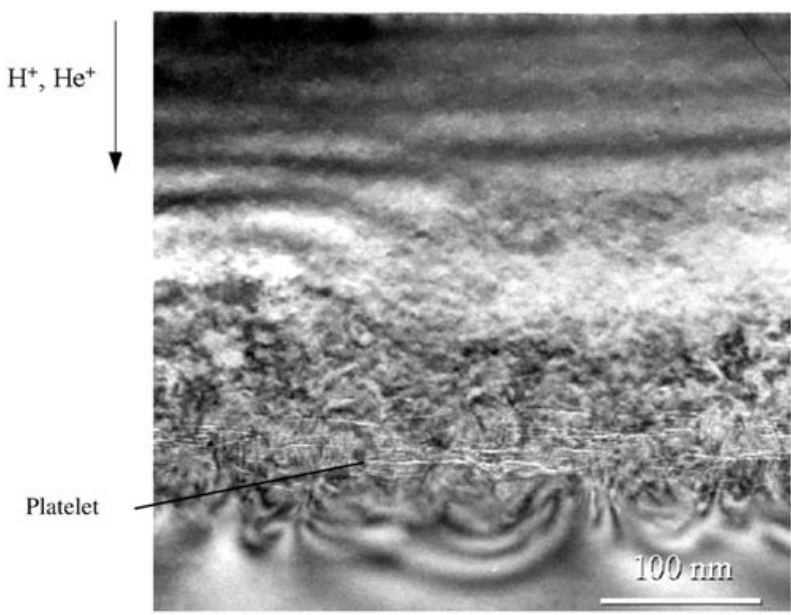

(b)

Figure 2. XTEM image of a sample annealed at $450{ }^{\circ} \mathrm{C}$

(a) Micro-cracks in silicon due to $\mathrm{H}^{+}$and $\mathrm{He}^{+}$implantation. The depth of the cracks is about $400 \mathrm{~nm}$ and should be the result of enlarged platelets connecting to each other. (b) Micro-cracks at the end of ion range. Most of the platelets are oriented along the $\{100\}$ plane. These platelets have not yet connected to each other and great strain is distributed near the platelet region. No bubbles can be found in either figure.

of the perpendicular strain component to the surface, $\varepsilon$, were obtained. This technique is very sensitive to lattice displacement and strain in the direction perpendicular to the working diffraction plane of the crystal.

$\mathrm{RBS} / \mathrm{C}$ were performed with $2.0 \mathrm{MeV} \mathrm{He}^{+}$ions. Rather than the raw RBS/C data, the profiles of the displaced atoms (or their integral) were calculated according to the algorithm developed in [2]. In the following, we shall consider the integral of the displaced atom profile as a quantity proportional to the intensity of the displacement field. HRXRD and channelling RBS can be considered as complementary techniques in order to detect the three-dimensional displacement field caused by point-like defects.

Samples for XTEM were prepared in the conventional way by mechanical thinning and $\mathrm{Ar}^{+}$ion etching. An XTEM observation concerns the distribution and location of extended defects, bubbles and platelets.

AFM was performed in either the contact or the tapping mode. The roughness values of the exfoliated and substrate surfaces were determined using a tapping mode with etched silicon tips. SEM was also performed.

The $\mathrm{H}$ distribution in the Si samples after implantation and annealing was measured by ERDA, using a $2 \mathrm{MeV}^{4} \mathrm{He}^{+}$beam (with a cross section of $1 \times 0.3 \mathrm{~mm}^{2}$ ) impinging the sample at 


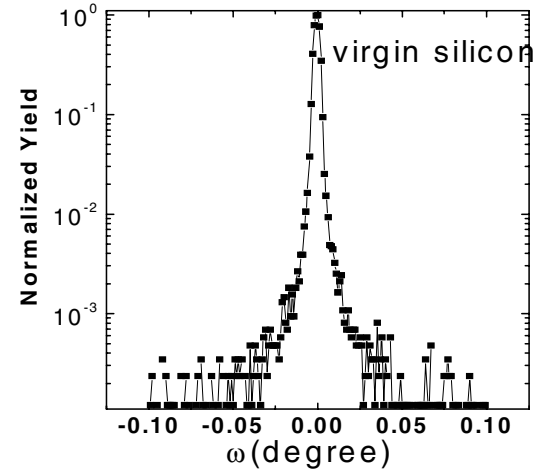

(i)

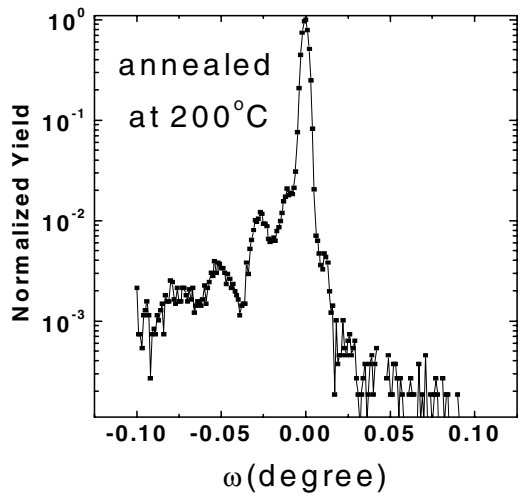

(iii)

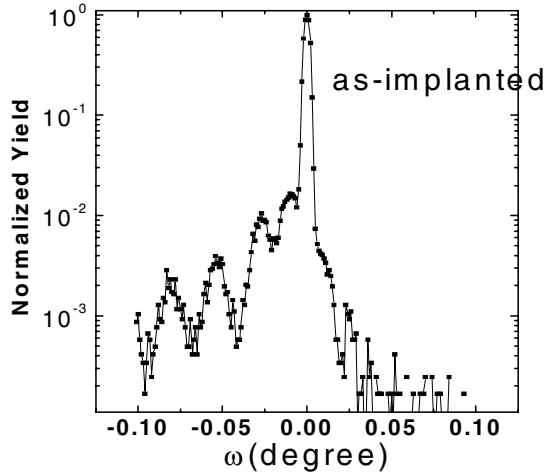

(ii)

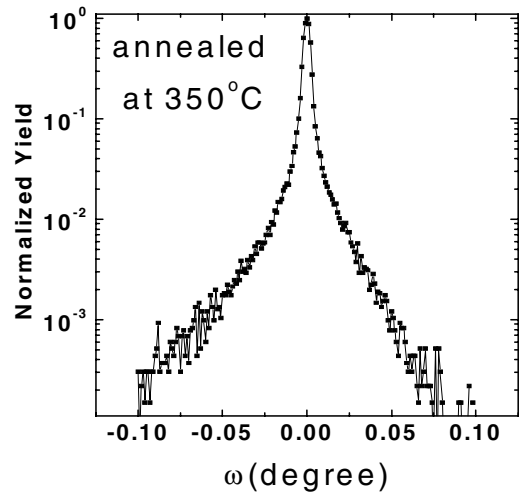

(iv)

(a)

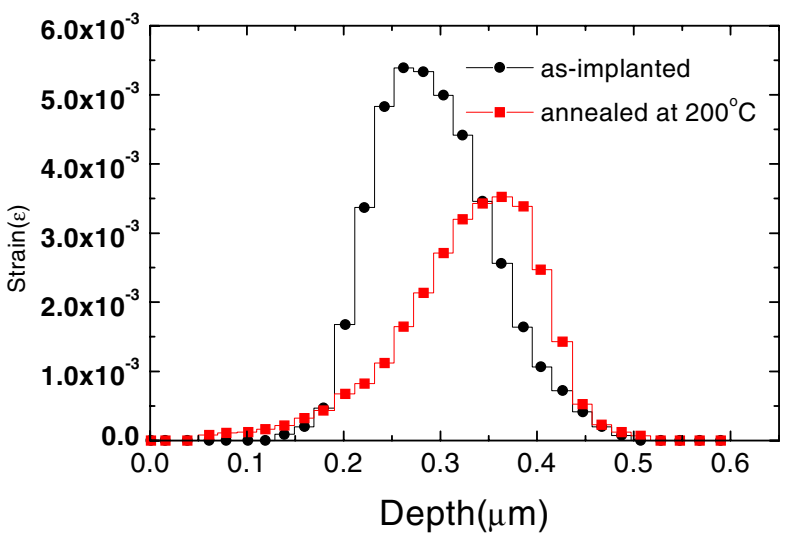

(b)

Figure 3. (a) The results of HRXRD. It is clear that after being annealed at $350^{\circ} \mathrm{C}$ the strain is much smaller, and the diffracted peaks from the strain disappear. (b) The distribution of strain in the samples annealed at $200{ }^{\circ} \mathrm{C}$ and the as-implanted samples. The distribution moves deeper after annealing, and become weaker and wider.

$75^{\circ}$ from the sample normal. The detector was placed at $150^{\circ}$ from the beam direction and the forward-scattered He particles were stopped by a $10 \mathrm{~mm}$ thick Mylar film.

\section{Results and discussion}

The internal crack formation on the overlying silicon was studied. AFM and SEM results of the samples annealed at
$450{ }^{\circ} \mathrm{C}$ are shown in figures $1(\mathrm{a})$ and (b). The depth of the craters measured by AFM is about $0.3 \mu \mathrm{m}$, which is equal to the projected range of implanted hydrogen. The area measured by AFM is only $5 \times 5 \mu \mathrm{m}^{2}$, so we cannot obtain a total image of a single blistering or an exfoliation crater. The SEM image shows an area of about $20 \times 20 \mu \mathrm{m}^{2}$. This confirms that the underlying cracks lead to a deformation of the overlying silicon. The presence of the capped bubbles on the Si surface 


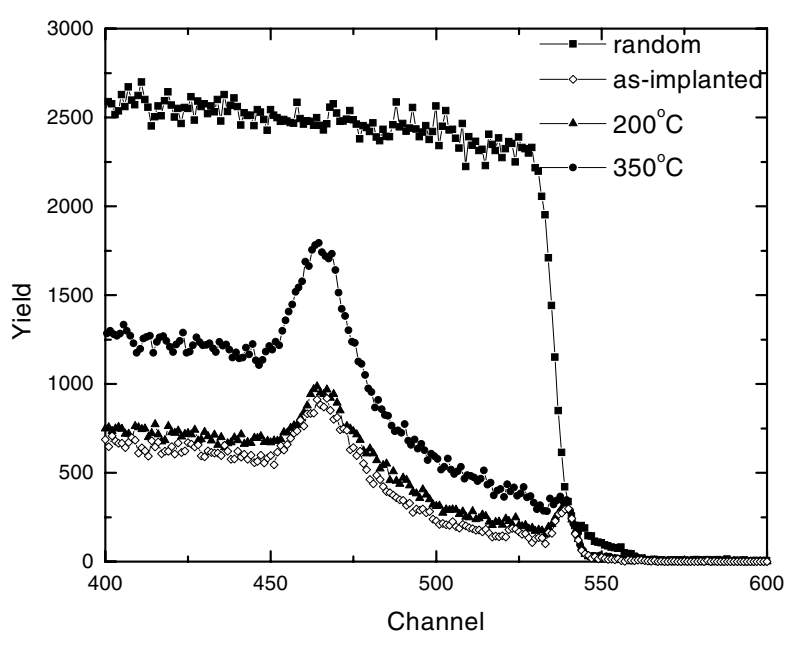

Figure 4. RBS/C spectra of the samples annealed at different temperatures.

strongly suggests that there is an internal pressure that causes elastic deformation of the overlying $\mathrm{Si}$.

XTEM was used to show the distribution of defects, platelets and bubbles in the samples. In figure 2, a typical electron micrograph of a thinner section reveals that most of the platelets in the defect layer are oriented along the (100) planes, but in crystal silicon implanted with hydrogen only the majority of the platelets are oriented along the (111) planes [1]. There are many dislocation loops and strains distributed near the implanted region. It is found that there are a few bubbles near the target area in the coimplanted samples, while there are many more bubbles in the hydrogenonly implanted samples.

To measure the strain distribution against depth, HRXRD was employed. Rocking curves of the samples annealed at different temperatures were obtained by HRXRD (shown in figure 3(a)). Ion implantation causes lattice swelling in the implantation region, and lattice swelling introduces strain in the overlying region. The rocking curves were computer simulated. The strain distribution against depth in the samples was obtained (shown in figure 3(b)). When the samples were annealed, the implanted region became amorphous. Consequently the unique determination of the local strain is no longer possible. So the rocking curves of the samples annealed at temperatures higher than $300^{\circ} \mathrm{C}$ were not computer simulated. We can see that after annealing at temperatures above $300{ }^{\circ} \mathrm{C}$ the strain in the lattice is cut down by nearly a half and the distribution of the strain becomes deeper and wider. This means that a large part of the hydrogen and the helium in the lattice escaped, and that they diffused deeper into the sample. In the temperature range from $300^{\circ} \mathrm{C}$ to $350^{\circ} \mathrm{C}$, scattering signals in the rocking curves increase rapidly. It is clear that there is great change in the samples annealed in this temperature range. In spite of the failure to simulate the rocking curves it is clear that the strain is much weaker here than in the samples annealed at $200^{\circ} \mathrm{C}$.

In the samples annealed in the temperature range of $200^{\circ} \mathrm{C}-450^{\circ} \mathrm{C}$, the scattering centre in the implanted region increases greatly. The distribution of the damage that occurs during the ion implantation and the thermal evolution of the

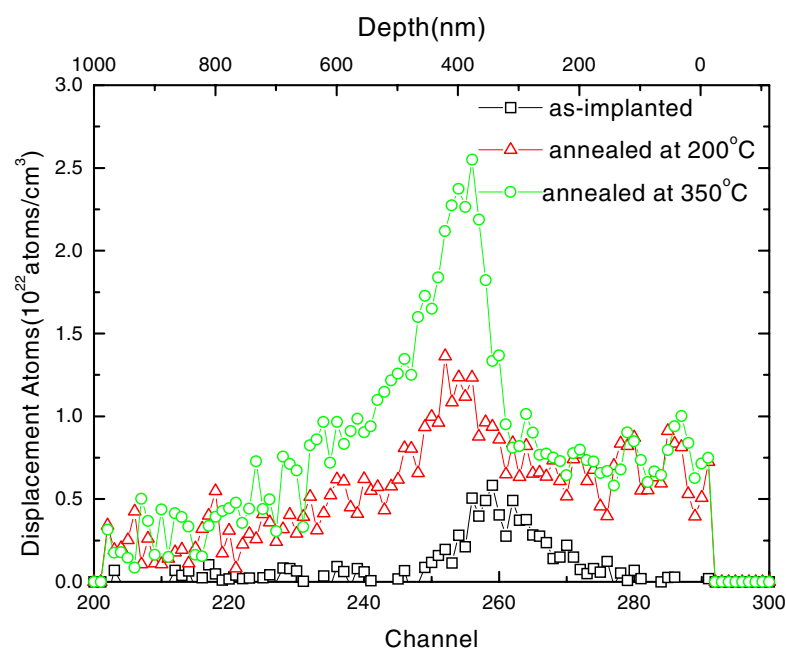

Figure 5. Displaced atom profiles of samples annealed at different temperatures. The density of the displaced atoms increases with the annealing temperature and the distribution moves deeper.

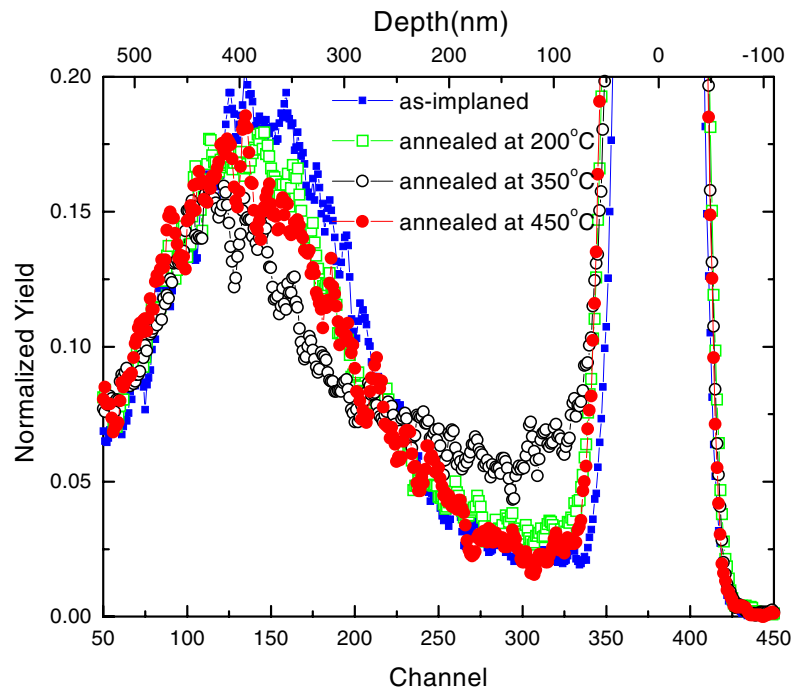

Figure 6. ERDA yield for samples annealed at different temperatures. The peak around channel 400 is due to contamination on the surface. The density of hydrogen decreases with the annealing temperature, but the distribution stays the same below $450^{\circ} \mathrm{C}$. The distribution of hydrogen in the samples annealed at $450^{\circ} \mathrm{C}$ changes considerably because exfoliation affects the measurements.

defects during annealing were investigated by RBS/C. Figure 4 shows the RBS/C spectra of samples annealed at different temperatures below $450^{\circ} \mathrm{C}$ for $1 \mathrm{~h}$. The channelling spectra of the samples indicate the density of the defects. When the samples were annealed at $450{ }^{\circ} \mathrm{C}$, the surface was blistered and exfoliated. A method described in [2] was used to analyse the RBS/C data and characterize the distribution of the displaced atoms. The distributions of displaced atoms against depth are presented in figure 5. The redistribution of hydrogen during annealing measured by ERDA is shown in figure 6. The as-implanted sample and samples annealed at $200{ }^{\circ} \mathrm{C}$ and $450^{\circ} \mathrm{C}$ have similar hydrogen distributions, but the concentration of hydrogen decreases with the increase of the annealing temperature, especially when the annealing 
temperature is $450^{\circ} \mathrm{C}$, at which point the samples exfoliate on the surface. The peaks around channel 370 are due to surface contamination.

When the implantation dose is not too high, we suppose that the magnitude of strain, $\varepsilon$, in the coimplanted region is in a direct ratio to the density of hydrogen and helium in the lattice (not hydrogen and helium in platelets or bubbles):

$$
\varepsilon=\alpha n_{H, L} N_{H}+\beta n_{H e, L} H_{H e} .
$$

In the above equation, $\alpha$ and $\beta$ are the strain parameters of hydrogen and helium, respectively; $n_{H, L}$ is the fraction of hydrogen in the lattice; and $n_{H e, L}$ is the fraction of helium in the lattice. Both $n_{H, L}$ and $n_{H e, L}$ are functions of the annealing temperature and the annealing time. $N_{H}$ and $N_{H e}$ are the density of hydrogen and helium in interstitial positions, respectively.

Hydrogen is a very reactive element. During implantation, a large part of the implanted hydrogen ions react with the silicon atoms or dangling bonds or defects, and form a diverse range of H-related complexes near the target area. Under certain conditions (e.g. annealed at high temperature), hydrogen atoms will be released from the $\mathrm{H}$-related complexes and combine with each other to form molecular hydrogen. Helium is an inert element. During implantation, helium atoms do not react with the silicon atoms or dangling bonds or defects and stay near the target. In general, for both elements, if they stay in the lattice the lattice will be enlarged and strain is introduced; if they stay in the platelets or bubbles they will not introduce strain into lattice. The hydrogen detected by ERDA is the hydrogen in all the components. The hydrogen in $\mathrm{H}-$ related complexes is bonded and difficult to move; molecular $\mathrm{H}_{2}$ and helium atoms are also difficult to move in the silicon lattice [1]; but the diffusivity of hydrogen atoms in silicon crystal is very high, and they will move away as soon as they are formed. Therefore, it is supposed that the hydrogen content detected by ERDA in the implantation region is the sum of molecular $\mathrm{H}_{2}$ and hydrogen in $\mathrm{H}$-related complexes. The density of hydrogen detected by ERDA is a direct ratio to the density of hydrogen.

During annealing, the hydrogen in the H-related complexes is released; some of it combines and forms molecular hydrogen in the lattice. The molecular hydrogen and the helium atoms have a low diffusivity and almost stay still in the silicon lattice at room temperature [1].

When annealed at about $200{ }^{\circ} \mathrm{C}$, the H-related complexes become unstable, collapse and release some hydrogen which forms molecular hydrogen. Both helium and molecular hydrogen are mobile and can diffuse into the near region. When the molecules remain in the lattice, the free energy is greater. They will diffuse into nearby platelets and bubbles, which does not change the size of lattice, and it was found that strain in these regions decreased. The diffusivity of molecular hydrogen and the helium atoms is not so high, so that they cannot diffuse far. Furthermore, the hydrogen bonds on the walls of the platelets and bubbles will also not enlarge the lattice. Therefore, the hydrogen and helium stay in the implanted regions, but they change their forms. In the simulation result of TRIM96, the distribution of implantation damage is not so deep as that of implanted hydrogen. In the damaged region, the density of dangling bond is much higher. The dangling bond will seize the free hydrogen atoms, which will stop the diffusion of the hydrogen atoms towards the surface. This leads to the hydrogen diffusing deeper.

In the temperature range from $300^{\circ} \mathrm{C}$ to $350{ }^{\circ} \mathrm{C}$ more hydrogen and helium in the lattice escaped away from lattice (based on the results of HRXRD). FTIR (Fourier transform infrared spectroscopy) [8] and RBS/C experiments on the annealing of hydrogen-implanted silicon also illustrate that there is some variation of lattice and chemical components in the hydrogen-implanted samples annealed in this temperature range. Many $\mathrm{VH}_{2}$ (where $\mathrm{V}$ denotes a vacancy) are generated [11]. The hydrogen and helium atoms greatly increase the free energy, so that the silicon atoms are 'kicked out' and form Frenkel pairs. The vacancies combine with the hydrogen. The silicon atoms become interstitial atoms, increasing the density of displacement atoms. The strain in the lattice decreases more. When annealed at $400^{\circ} \mathrm{C}$ or higher, the pressure of the gas in the platelets leads to the exfoliation of the surface, $\mathrm{H}_{2}$ and helium atoms release from the silicon crystal. The density of both hydrogen and helium in the silicon decreases significantly. During the process of exfoliation, defects are generated in great quantities. Defects and blisters on the surface of the silicon substrate cause the significant increase of RBS/C yield. In the following we will discuss the processes of blistering and exfoliation during annealing discussed in this section.

The role of implanted $\mathrm{H}$ is twofold; first, it acts chemically, in that it drives the formation of microscopically flat internal surfaces-platelets - and acts as a source of gaseous $\mathrm{H}_{2}$ which is trapped in the internal cavities; second, it acts physically, as an internal pressure source. During hydrogen ion implantation, extended defect platelets, bubbles and vacancies are generated. The inner surfaces of these extended defects are hydrogenized. Some of the $\mathrm{Si}-\mathrm{Si}$ bonds are replaced by $\mathrm{Si}-\mathrm{H}-\mathrm{H}-\mathrm{Si}$ bonds (one of the most important H-related complexes that causes exfoliation) which are much weaker than the Si-Si bonds [1]. During annealing, some of the H-related complexes relapse, and molecular hydrogen is formed in the silicon crystal. The breaking of hydrogen bonds in $\mathrm{Si}-\mathrm{H}-\mathrm{H}-\mathrm{Si}$ introduces platelets. If the molecular $\mathrm{H}_{2}$ stays in the platelets, the free energy of the system is much lower than that when they remain in interstitial sites. So molecular $\mathrm{H}_{2}$ is apt to accumulate in these extended defects, and exerts great pressures on the inner surfaces of the extended defects. The structure of the platelets is easier to split than other extended defects due to their mechanical and chemical structure. During annealing, $\mathrm{H}_{2}$ molecules and helium atoms are released from the lattice and accumulate in the platelets. The platelets become larger and larger under the internal pressure; finally some of them connect with each other. The diameters of the platelets are several nanometres before annealing, and several tens of nanometres after annealing [8]. When the annealing temperature is above $400{ }^{\circ} \mathrm{C}$, blistering and exfoliation occurs. During the splitting process, platelets play a much more important role than the bubbles. The above processes are shown in figure 7.

Based on the model of exfoliation stated above, the relation of hydrogen/helium implantation total dose and the fraction of hydrogen in implantation is calculated. 


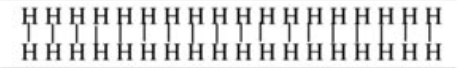

(a)

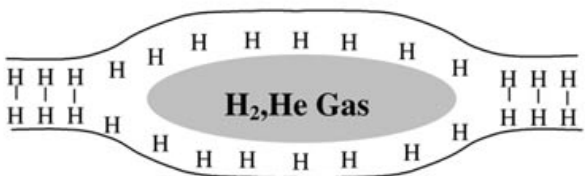

(b)

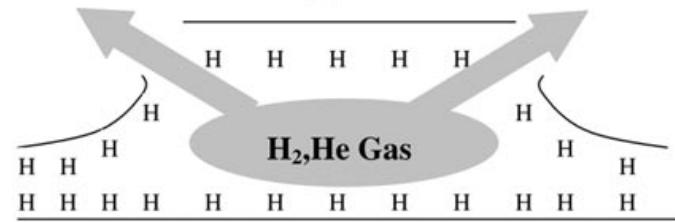

(c)

Figure 7. The evolution process from H-related complexes to platelets. (a) As-implanted, H-related complexes form. (b) A platelet comes into being, and gas accumulates in it during annealing. (c) The surface exfoliates under the great gas pressure when annealed at high temperatures.

We divide all of the hydrogen and helium in the silicon into two forms, the component in the lattice and the component in the platelets. We suppose the average free energy of the hydrogen in the lattice is $E_{H, I}$, the average free energy of the hydrogen in the platelets is $E_{H, P}$, the average free energy of the helium in the lattice is $E_{H e, I}$ and the average free energy of the helium in the platelets is $E_{H e, P} . \quad D_{H}$ and $D_{H e}$ are the implantation densities of the hydrogen and the helium, respectively. $\rho_{s}$ is the surface density of hydrogen atoms and $f(T)$ is the thermodynamic fraction that comes off the walls. $\gamma$ is the surface energy of the walls in the platelets. $r_{0}$ is the average diameter of the platelets. $N_{0}$ is the average number of gas molecules in the platelets. $N_{\text {platelet }}$ is the density of platelets. $N_{H, P}$ and $N_{H e, P}$ are the densities of the hydrogen and helium atoms in bubbles or platelets, respectively. $r_{e x}$ is the average radius of the platelets when they connect to each other during annealing. According to our experimental results, the temperature $T_{e x}$, at which the surface exfoliated during annealing, is almost the same in the different implantation dose samples. We obtain the following formulae.

$$
\begin{gathered}
\frac{N_{H, P}}{N_{H, I}}=\frac{\mathrm{e}^{-E_{H, P} / k_{B} T}}{\mathrm{e}^{-E_{H, I} / k_{B} T}} \\
\frac{N_{H e, P}}{N_{H e, I}}=\frac{\mathrm{e}^{-E_{H e, P} / k_{B} T}}{\mathrm{e}^{-E_{H e, I} / k_{B} T} .}
\end{gathered}
$$

Equations (2) and (3) give the relationship between the number of gas atoms in the different states under a Boltzmann distribution:

$$
\begin{gathered}
N_{\text {platelet }}=C N_{H, I} \\
r_{0}^{2}=\frac{1}{4 \pi} \frac{3 k_{B} T N_{0}}{\gamma-\frac{4}{3} \rho_{s} k_{B} T f(T) \ln \left(3 T N_{0} / 4 \pi \gamma\right)} .
\end{gathered}
$$

Equation (5), cited from [10], gives the relationship between the radius of the platelets and the annealing temperature and the number of gas molecules in the platelet.

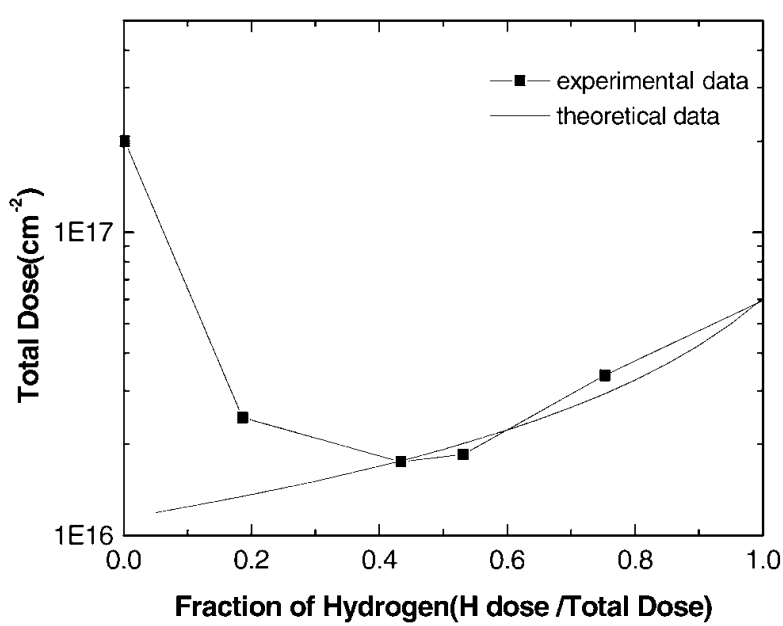

Figure 8. The experimental data (from [10]) and the theoretical curve of the total $\left(\mathrm{H}^{+}+\mathrm{He}^{+}\right)$implantation dose necessary for blistering as a function of the fraction of $\mathrm{H}^{+}$.

To simplify the calculation, we assume that the all the platelets are at the same depth and parallel to the surface:

$$
\begin{gathered}
N_{\text {platelet }} r_{e x}^{2}=1 \\
N_{0}=\frac{\left(\frac{1}{2} N_{H, P}+N_{H e, P}\right)}{N_{\text {platelets }}} .
\end{gathered}
$$

Equation 6 is the condition when the platelets connect to each other; $r_{0}>r_{e x}$ is the condition of exfoliation. Equation 7 gives the average number of gas molecules in one platelet.

We assume that when $r_{0}>r_{e x}$, the surface will exfoliate. By combining the above formulae, we can see that

$$
\frac{\alpha D_{H}+\beta D_{H e}}{\gamma-\delta \ln \left(\left(\alpha D_{H}+\beta D_{H e}\right) / k_{B} \gamma N_{\text {platelets }}^{2}\right)} \geqslant 1
$$

where $\alpha, \beta, \delta$ are constants derived from the above formulae:

$$
\begin{gathered}
\alpha=\frac{3 k_{B} T_{e x}}{8 \pi\left(1+\exp \left(\left(E_{H, P}-E_{H, T}\right) / k_{B} T_{e x} N_{\text {platelets }}\right)\right.} \\
\beta=\frac{3 k_{B} T_{e x}}{4 \pi\left(1+\exp \left(\left(E_{H, P}-E_{H, T}\right) / k_{B} T_{e x} N_{\text {platelets }}\right)\right.} \\
\delta=\frac{4}{3} \rho_{s} k_{B} T_{e x} f\left(T_{e x}\right) .
\end{gathered}
$$

The radius of the platelets is quite small under the above hypothesis; according to [10], we can conclude that $\gamma \gg$ $\delta \ln \left(\left(\alpha D_{H}+\beta D_{H e}\right) / k_{B} \gamma N_{\text {platelets }}^{2}\right)$, and we obtain

$$
\alpha D_{H}+\beta D_{H e} \geqslant \gamma \text {. }
$$

Compared with the experimental data [10], if one only implants hydrogen the minimum dose required is $6 \times$ $10^{16} \mathrm{~cm}^{-2}$, but, when coimplanting hydrogen and helium, if the hydrogen implantation dose is $0.75 \times 10^{16} \mathrm{~cm}^{-2}$ then the helium minimum implantation dose is about $1 \times 10^{16} \mathrm{~cm}^{-2}$, and we find that $\alpha / \gamma=1.67 \times 10^{-17} \mathrm{~cm}^{-2}$ and $\beta / \gamma=$ $8.75 \times 10^{-17} \mathrm{~cm}^{-2}$. The theoretical curve fits the experimental curves very well when the hydrogen implantation dose is not too low. The result is shown in figure 8 . 
When only helium ions are implanted or the content of hydrogen is very low, the situation is quite different. Because of the absence of any hydrogen being introduced into the platelets, the surface exfoliation is caused only by the pressure in the bubbles, which is a much more difficult way to cause exfoliation than by first forming platelets. Therefore it will not be discussed here.

\section{Conclusion}

In this paper we found that coimplantation did not affect the annealing temperature of surface exfoliation. The variations of the strain and the defects during annealing in the temperature range from $200^{\circ} \mathrm{C}$ to $450{ }^{\circ} \mathrm{C}$ were analysed. We concluded the following.

(1) In the as-implanted $\mathrm{Si}$, a damage region is formed around the target area, and the damage became greater during annealing at temperatures below $450{ }^{\circ} \mathrm{C}$.

(2) During annealing, part of the implanted hydrogen and helium convert into the components that do not contribute to strain in the samples; we ascribe the component to the $\mathrm{H}_{2}$ (molecular) and helium in bubbles and platelets.

(3) When the annealing temperature is low, hydrogen and helium are very slowly released, but when the annealing temperature is at about $450{ }^{\circ} \mathrm{C}, \mathrm{H}_{2}$ and helium gas are released much more rapidly.

\section{Acknowledgments}

This work was supported by the National Natural Science Foundation of China (No 19775062 and No 69976034) and the Shanghai Youth Foundation (No 98JC14004).

\section{References}

[1] Pearton S J, Corbett J W and Stavola M 1992 Hydrogen in Crystalline Semiconductors (Berlin: Springer) and references therein

[2] Pankove J I and Johnson N M (eds) 1990 Hydrogen in Semiconductors (New York: Academic)

[3] Heinrich H and Jantsch W (eds) 1993 Defects in Semiconductors 17 (Materials Science Forum) (Switzerland: Trans Tech.) pp 143-7

[4] Wong-Leung J, Ascheron C E and Petravic M 1995 Appl. Phys. Lett. 661231

[5] Wong-Leung J, Nygren E and Williams J S 1995 Appl. Phys. Lett. 67416

[6] Wong-Leung J et al 1995 Nucl. Instrum. Methods B 96253

[7] Bruel M 1995 Electron. Lett. 311201

[8] Weldon M K et al 1997 J. Vac. Sci. Technol. B 151065

[9] Agarwal A, Haynes T E, Venezia V C, Holland O W and Eaglesham D J 1998 Appl. Phys. Lett. 721086

[10] Varma C M 1997 Appl. Phys. Lett. 713519

[11] Weldon M K, Collot M, Chabal Y J, Venezia V C, Agarwal A, Haynes T E, Eaglesham D J, Christman S B and Chaban E E 1998 Appl. Phys. Lett. 733721 